remaining 5 presented with unusually mild or atypical symptoms, suggesting a peroxisome biogenesis disorder, with variable expression in different tissues. Clinical symptoms vary with age: neonatal- hypotonia, seizures, dysmorphisms, and skeletal abnormalities; 1-6 months-failure to thrive, hepatomegaly, jaudice, retinopathy, cataract; 6 months - 4 years- neurological presentation, retardation, visual and hearing impairment, osteoporosis; beyond 4 years- behavior changes, intellectual deterioration, leukoencephalopathy, peripheral neuropathy. Assay of plasma very long chain fatty acids (VLCFAs) is a general screening test for peroxisomal disorders, but VLCFA elevation may be small in mild variant patients. Diagnostic assays should include plasma phytanic, pristanic, and docosahexaenoic acids; urine organic acids and pipecolic acid; red blood cell plasmalogens; fibroblast plasmalogen synthesis; and liver cytochemical localization of peroxisomal proteins. The diagnostic usefulness of pipecolic acid measured on routine amino acid chromotography is emphasized, especially in atypical cases. (Baumgartner MR, Poll-The BT, Verhoeven NM, et al. Clinical approach to inherited peroxisomal disorders: a series of 27 patients. Ann Neurol Nov 1998;44:720-730). (Respond: Dr JM Saudubray, Department of Pediatrics, Hopital NeckerEnfants Malades, Paris Cedex 15, France).

COMMENT. Peroxisomal disorders may present in a variety of clinical manifestations, often age-dependent. The most common peroxisomal disorder, $\mathrm{X}$ adrenoleukodystrophy (X-ALD), may present with hyperactivity and attention disorders. According to an editorial commentary, the diagnosis of X-ALD should be suspected when ADHD is complicated by dementia, incoordination, or auditory or visual impairment (Moser HW, Raymond GV. Genetic peroxisomal disorders: why, when, and how to test. Ann Neurol Nov 1998;44:713-715). The demonstration of abnormally high levels of VLCFAs in plasma is the prototype initial test. Studies of cultured skin fibroblasts are the most helpful additional test. Other diagnostic assays, including immunochemical assays, are necessary in diagnosis of atypical cases. Diagnosis is important in genetic counseling and disease prevention.

\title{
NEUROLEPTIC MALIGNANT SYNDROME AND METHYLPHENIDATE
}

Neuroleptic malignant syndrome probably caused by methylphenidate (MPH) is reported in a 1-year-old female infant treated at the Institute of Neurological Sciences, Tottori University Faculty of Medicine, Yonago, Japan. Born with hypoxic-ischemic-encephalopathy (HIE) and multicystic encephalomalacia, she was treated with MPH (3 mg/day) at 1 year, 6 months of age, because her circadian rhythm was reversed and irregular. One day after starting MPH, she developed otitis media that resolved with amoxicillin in 5 days. An abrupt onset of fever $\left(39.7^{\star} \mathrm{C}\right)$ after 6 days was associated with muscular rigidity. A CK level of $17,205 \mathrm{IU} / \mathrm{L}$ on admission gradually fell to 579 by the 8 th day. The HIE, infection, and vegetative state were possible predisposing factors. The pathogenesis and dopaminergic blockade mechanism of the syndrome is discussed. (Ehara H, Maegaki Y, Takeshita K. Neuroleptic malignant syndrome and methylphenidate. Pediatr Neurol Oct 1998;19:299-301). (Respond: Dr Ehara, Department of Pediatrics, Western Shimane Medical Center for the Handicapped, 1926 Watazu-cho, Gontsu 695-0001, Japan).

COMMENT. The three major manifestations of neuroleptic malignant syndrome (NMS) are fever, rigidity, and elevated CK level. Additional characteristic symptoms include tachycardia, tachypnea, altered consciousness, and leukocytosis. Severe brain damage due to HIE and otitis media might be predisposing factors. This is the first reported case of MPH-induced NMS. 